\title{
Subclinical Ptosis Correction: Incision, Partial Incision, and Nonincision: The Formation of the Double Fold
}

\author{
Yong Kyu Kim, MD, $\mathrm{PhD}^{1}$ Abdulla Fakhro, MD ${ }^{2}$ Anh H. Nguyen, MD ${ }^{2}$
}

${ }^{1}$ Apgujeong YK Plastic Surgery Clinic, Seoul, Korea

${ }^{2}$ Division of Plastic Surgery, Baylor College of Medicine, Houston, Texas

Address for correspondence Yong Kyu Kim, MD, PhD, Apgujeong YK Plastic Surgery Clinic, Seoul, Korea (e-mail: psinbrain@gmail.com).

Semin Plast Surg 2015;29:165-170.

\begin{abstract}
Keywords

- subclinical ptosis

- levator aponeurosisMüller muscle complex

- double eyelids

Surgery to create eyelid folds accounts for the highest percentage of surgeries in Asians and Koreans who receive the surgery on the upper eyelid 2 to 3 times during their lifetimes for functional or cosmetic reasons. Patients are generally satisfied with the results-the eyes becoming brighter and bigger via the improvement of pseudoptosis by fold creation. The recent trend is to seek the "perfect" eye: a vertically and horizontally big palpebral fissure with more than $90 \%$ cornea showing. Surgery of the levator aponeurosis-Müller muscle complex is required to expose the cornea, except in those patients who inherently have good levator-Müller function. However, many complications occur during surgeries of the levator aponeurosis-Müller muscle complex, which increase the reoperation rate. Here, the authors briefly summarize recent experiences correcting subclinical ptosis using the nonincision, incision, and partial incision methods.
\end{abstract}

Good levator aponeurosis-Müller muscle complex function is one of the most important factors in the creation of cosmetically beautiful double eyelids. However, other factors require consideration in the creation of cosmetically beautiful eyes, including the thick skin and muscle involved, positions of the fat and eyebrows, correlation between the eyes and the maxillary infraorbital rim, and the presence or absence of a slant Mongolian fold. ${ }^{1}$

Ptosis refers to visual discomfort that occurs when the levator aponeurosis-Müller muscle complex function is weak. In contrast, pseudoptosis refers to a condition in which the eyes are obstructed by drooping skin despite normal muscle function. In the latter case, if the skin is surgically folded once or excised, corneal exposure is increased, which makes the eyes appear bigger and brighter. Most patients who desire double eyelids have pseudoptosis, so if the skin is simply incised and fixed to tarsal plate, or a fold is made using the nonincision method, the results are generally satisfactory. ${ }^{1-3}$

However, based on our experience, despite the appearance of a fold after surgery, approximately $30 \%$ of patients still feel that their eyes look small and swollen. Over time, the double eyelids become shallow or disappear due to weak levator aponeurosis-Müller muscle complex function. ${ }^{4,5}$ These cases are called subclinical ptosis, which can be identified before surgery if patients say "I always look sleepy," "I open my eyes with my forehead wrinkled," "My eyes feel tired in the afternoon," or "If I don't open my eyes widely when pictures are taken, then they look closed in pictures." Objectively, if the cornea is blocked superiorly by $>2 \mathrm{~mm}$ in younger patients, they can be diagnosed with subclinical ptosis. ${ }^{5}$ However, it is not pathological, and if there is no patient desire for correction of this nonpathological, subclinical ptosis, surgery cannot be recommended based on numeric measurement values only. In fact, the distance between the eye and brow, and the shape of brow are also important variables in a beautiful eye. Some patients who have subclinical ptosis and many layers of shallow folds or asymmetrical double eyelids have attractive eyes, which can be attributed to a slightly higher position of the eyebrows, an enhancement of the femininity of the face relative to the vertical proportion of thirds. ${ }^{6}$
Issue Theme Cosmetic Asian Blepharoplasty and Periorbital Surgery; Guest Editor, Anh H. Nguyen, MD 


\section{Hering's Law of Equal Innervation: Binocular Coordination of Eye Movements}

The correction of asymmetry is the most popular goal that patients mention before surgery. However, this is also the most common postsurgical complaint despite efforts to provide informed consent prior to the surgery. A slight difference in fold heights becomes equal over time in many cases, but subclinical ptosis never improves without intervention. When a surgeon is correcting asymmetric ptosis, one factor to consider is Hering's law of equal innervation.?

If the patient shown in $\mathbf{- F i g .} \mathbf{1}$ requested correction of both eyes to create a symmetrical look, on which side of the eyes would you conduct the surgery? This patient appears to have ptosis in the left eye and normal levator aponeurosisMüller muscle complex function in the right eye. However, if each eye is examined individually, the ptosis of the left eye appears more severe and a low degree of ptosis is observed in the left eye (Hering's law).

In this case, if you think the right eye is identified as normal, levator aponeurosis-Müller muscle complex surgery is performed on the left eye without consideration of Hering's law; after surgery, the left eyelid goes upward and the right eyelid goes down and looks sleepy, so a secondary operation cannot be avoided.

In conclusion, this patient requires surgery in both eyes because asymmetry is caused by differing degrees of levator aponeurosis-Müller muscle function. If the heights of the folds are made equal, cosmetically beautiful double eyelids can be obtained after gaining of symmetry of levator function by ptosis surgery.

\section{YK Test: Guideline for Using the Incision versus Nonincision Method}

When patients with subclinical ptosis present to the clinic, if the presence/absence of ptosis and the presence/absence of symmetry in the levator aponeurosis-Müller muscle complex function have been identified, the surgical method can be chosen.

According to a classically selected method, the orbital septum is opened after the skin incision, the levator aponeurosis-Müller muscle complex is resected or plicated, and a fold is made by tarso-dermal fixation. ${ }^{8}$ In contrast, with the increasingly popular nonincision method, the levator aponeurosis-Müller muscle complex function is corrected

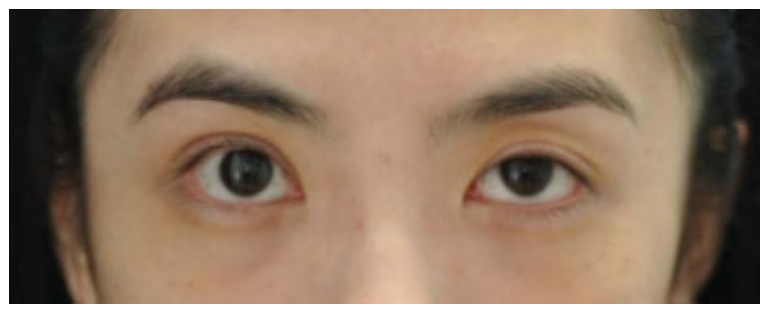

Fig. 1 Hering's law: Asymmetric ptosis. through the conjunctiva and a fold is made by conjunctivolevator-skin sling. ${ }^{9}$

Choosing the appropriate method is problematic for every surgeon. If the same outcome is expected, it is reasonable to select a less-invasive method that features less swelling, a quick recovery, and fewer complications.

We have had the same problem when treating patients and have found a good strategy for choosing the right surgical technique between the incision and nonincision methods.

1. The surgeon and the patient directly face each other while seated.

2. Using a wire crease maker, the surgeon places a spot 6 to $7 \mathrm{~mm}$ above from the patient's upper eyelashes and says "open your eyes" while moving the wire crease maker upward (-Fig. 2).

3. At this time, if the eyelid moves up smoothly, exposure of the cornea becomes better, and the double eyelid lines appear clearly; surgery should be conducted using the nonincision method. In contrast, if resistance is felt and the eyelids become everted, then scar tissue or fibrosis is present in the orbital septum and the levator aponeurosis glide plane; surgery should be conducted using the incision method.

Despite the feeling of resistance, if it does not seem severe, the partial incision method can be used. In such cases, the patient is informed in advance that they will be asked to open their eyes during the surgery and that the method may be changed as to the incision method as needed to achieve the desired outcome.

\section{Anatomy}

There are stark differences between the Asian eyelid and Caucasian eyelid. First, only a small percentage of Asians have an evident supratarsal fold. Moreover, there often is an absent or low supratarsal fold with prominent periorbital tissues. ${ }^{3,10}$

The point of fusion of the orbital septum with the levator aponeurosis governs the location of the supratarsal fold. ${ }^{3}$ It is at that location that the levator aponeurosis sends fibers to the overlying skin, anchoring it down to the eyelid and creating the lid fold. In the Asian eyelid, this fusion between the septum and the levator aponeurosis is often low, making the supratarsal fold seem closer to the eyelid margin. ${ }^{11}$ This fusion can be as low as the lower anterior portion of the tarsus near the lid margin. Because the septal-levator fusion is lower

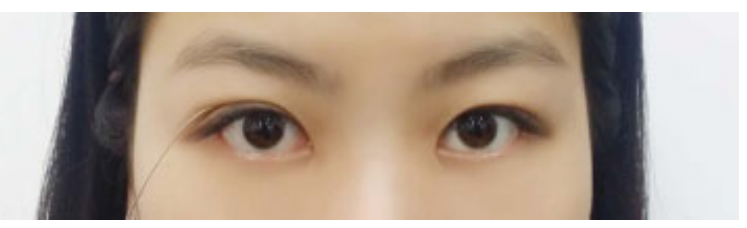

Fig. 2 Wire crease maker located 6 to $7 \mathrm{~mm}$ from eyelash and gently moving upward with eye opening. This patient opened her eye more on the medial side without eyelid eversion. In this case, a nonincision technique is the appropriate procedure. 
on the eyelid, the retroseptal fat descends in the eyelid and yields a fuller and thicker eyelid ( - Fig. $\mathbf{3}$ ). ${ }^{12}$

In addition, the Asian eyelid often has excess fatty tissue, a narrow palpebral fissure, downward pointing eyelashes without a cutaneous insertion to the levator muscle, and the superior palpebral fold is absent. Epicanthal folds are present in about half the Asian population. ${ }^{13}$

In some subclinical ptosis cases, the levator muscle is very thin and transparent; this means that only the Müller muscle elevates the eyelid-even Müller muscle also detached from the tarsal plate in the medial region. This finding is noted often in the exophthalmic eye patient. This make sharp triangular shape of palpebral fissure and looks narrower (-Fig.4).

A detailed description of the anatomy of the Asian eyelid is provided in "Asian Eyelid: Relevant Anatomy" by Kiranantawat et al in this issue.

\section{Neurophysiology}

As with breathing, our eyes function naturally without the need for conscious thought. In contrast, deep breathing is an intentional movement, as are the voluntary movements of widely opening or tightly closing the eyes.

A

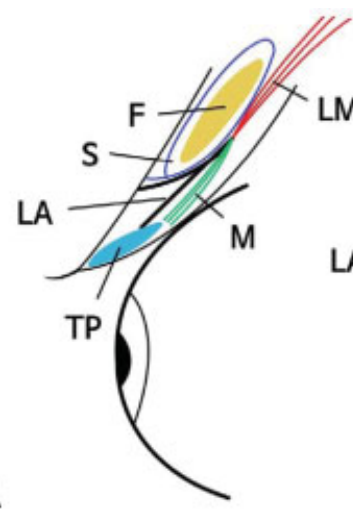

The levator muscle is responsible for the involuntary movements of the eyes, whereas the Müller muscle controls voluntary movements. ${ }^{14}$ In addition, the Müller muscle recognizes stretching of the levator muscle during eye closure and sends feedback to the mesencephalic trigeminal nucleus, leading to autonomic opening of the eyes. ${ }^{14}$

In most patients with subclinical ptosis, the feedback mechanism does not work normally because either the muscles are not fully developed congenitally or mechanical problems of dehiscence or malinsertion exist in the levator aponeurosis-Müller muscle complex, which leads to ptosis. Based on our experience, even in the presence of little muscle fiber content, correcting ptosis is not problematic. However, there was anatomical weakness or attenuation between the levator aponeurosis-Müller muscle complex and tarsal plate in most of our cases, so this part we completely removed and then advanced together during the reconstruction, leading to function again as a stretch receptor. This resulted in satisfactory outcomes only with 4 to $6 \mathrm{~mm}$ of advancement in most cases, which also avoided lagophthalmos by excessive advancement.

\section{Nonincision Müller Tucking Method}

Just as surgical methods have improved from incision to endoscopic and robotic surgery, the surgery for ptosis correction and creating folds has improved from the incision method to the nonincision method. These changes were motivated by an understanding of anatomy and neurophysiology. It is generally known that if a fold is made without an incision, it is likely to disappear. ${ }^{15}$ However, despite several reports on the disappearance rate, there have been few reports on its related causes and measurements. The cheese-cutting effect is the term used to describe what happens when the skin is sutured to the tarsal plate or the weak levator aponeurosis-Müller muscle complex using 7-0 nylon. Even in the incision method, fold is initially fixed well by 7-0 nylon; nevertheless, over time, the thread is loosened off from the tarsal plate and levator aponeurosis, and erodes into the skin because of continuous traction power, resulting in fold disappearance. ${ }^{16}$ This is similar to the scenario that if a nylon thread moves in and out of cheese, the cheese is cut while the thread remains intact. In the nonincision Müller tucking method, the Müller muscle is approached through conjunctiva, followed by the tucking of 6 to $10 \mathrm{~mm}$ to rebuild neurophysiological feedback. If the part that used to be loose is strengthened by tucking and the skin is sutured with 7-0 nylon to make the fold, the chance of the cheese-cutting effect decreases to maintain a semipermanent fold similar to that of the incision method (- Fig. 5) ${ }^{9}, 17$

\section{Incision Method}

The incision method can be divided into two methods depending on the anatomy that fixes the skin to make the fold: one is the tarsal plate and another is the levator aponeurosis-orbital septum complex. The latter is a dynamic method, but adjusting symmetry is inconvenient because fold
Fig. 4 Blue coloration indicates the Müller muscle. On the medial side, Müller muscle is not developed or there is dehiscence on right eye and the left side Müller muscle is well developed. During surgery, the surgeon has to find any existing pathology in the Müller-tarsal plate and correct it. 


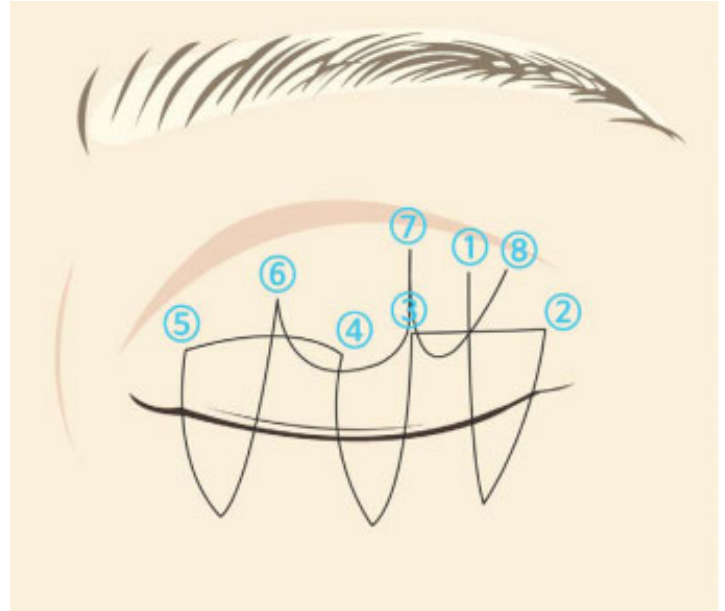

Fig. 5 Nonincision single-stitch continuous suture with transconjunctival Müller tucking method: A 6- to 10-mm Müller tucking is performed.

depth and height change with levator aponeurosis muscle function. Most patients have satisfactory outcomes regardless of the fixation method if the levator aponeurosis muscle has good functionality. In contrast, in cases of subclinical ptosis, the ptosis must first be corrected symmetrically by correction of the levator aponeurosis-Müller muscle complex in both eyes followed by fixation of the fold heights to the tarsal plate. The use of this method enables predictable outcomes. ${ }^{17,18}$

The incision method is indicated in cases that have a lot of fibrosis and scar tissue determined by the YK test. Factors interfering with gliding are first removed, the levator aponeurosis-Müller muscle complex is completely separated from the tarsal plate, and the levator aponeurosis muscle is also separated from Müller muscle by approximately 4 to $5 \mathrm{~mm}$. These steps are performed to restructure the feedback mechanism. In this state, the levator aponeurosis-Müller

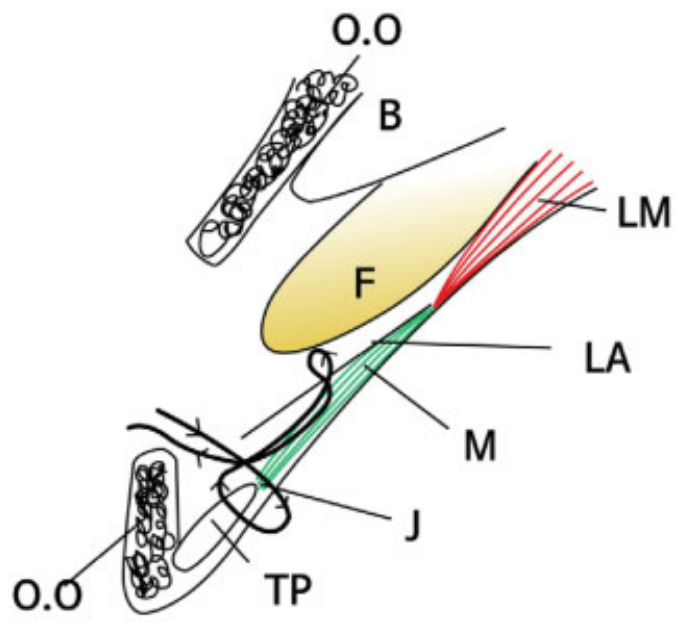

Fig. 6 Incision method with Levator-Müller advance using a figure 8 suture. A 4- to 6-mm advance of levator-Müller complex is enough for rebuilding neurophysiological feedback. B, bone; F, orbital fat; J, junction between cartilage and aponeurosis; LA, levator aponeurosis; LM, levator muscle; M, Müller muscle; TP, tarsal plate. muscle complex is advanced to within the 4- to 8-mm range for fixation to the tarsal plate and the patient is asked to open their eyes so the surgeon can check the degree of correction. In general, after fixation of both sides using a single point is performed, the patient is asked to open their eyes for comparison. If a large degree of swelling occurs during surgery, it is difficult to compare the eyes, so careful hemostasis and control are required during surgery. Single-point fixation to 1 to $2 \mathrm{~mm}$ lateral from the middle and fixation at the medial limbus are usually sufficient. Based on our experiences, because the medial portion of the Müller muscle is rarely developed in Asians, if fixation is not performed in the medial limbus area, the shape of medial side is not beautiful. Accordingly, fixation of 5 to 6 points of skin and muscle to the tarsal plate is required; if fewer points are used, the fold may be disappeared as time goes by. ${ }^{17,18}$

The last step is skin suture; we think that skin-to-skin suture is enough for keeping a fold, although some surgeons recommend skin-to-tarsal-plate-to-skin suture during skin suture (-Fig. 6).

\section{Partial Incision Method with Müller Tucking and Orbital Fat Release}

The main goal of the partial incision method is not to remove fat or advance the levator aponeurosis-Müller muscle complex through it, but to entirely release the septum in 360 degrees, which is surrounding orbital fat, to remove anatomy that interferes with gliding between the posterior septum such as the conjoined tendon or fibrosis and the levator aponeurosis and to remove the fat that naturally flows out after releasing and then it could be removed or preserved in sunken case ( - Fig. 7). In most patients with subclinical ptosis, fat in the orbital septum located higher than normal patients, so that while the eyes are open, they appear sunken in many
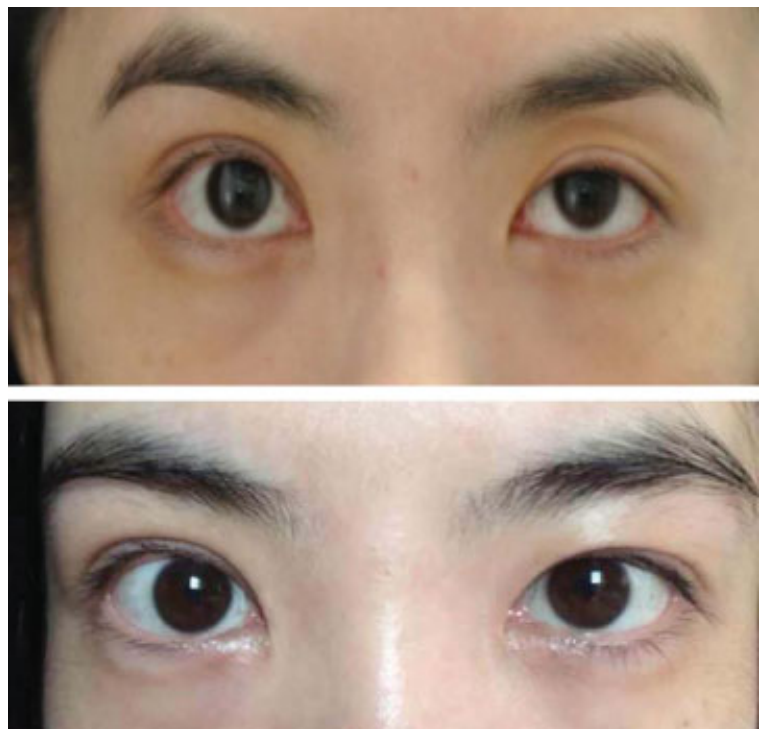

Fig. 7 A patient from China who suffered asymmetry after blepharoplasty. Asymmetry and sunken eyelid is corrected after levatorMüller surgery. 


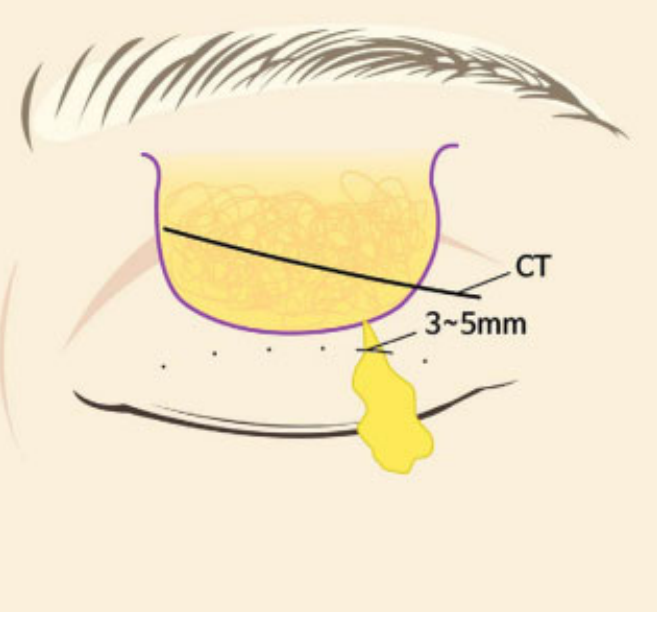

Fig. 8 Conjoined tendon cutting, fibrotic septum releasing, and fat removal is possible through 3- to 5 -mm-step incision. $\mathrm{CT}$, conjoined tendon.

cases. As a result, if the fat is completely released through a partial incision and the Müller muscle is tucked toward the conjunctiva, ptosis and the sunken eye also are corrected (-Fig. 8). . $^{9} 19$

\section{Complications}

\section{Fold Disappearance}

Fold disappearance is a relatively common complication of the nonincision and incision methods in subclinical ptosis patients. To prevent fold disappearance as mentioned before, it is better to create the fold by separating the ptosis correction with levator aponeurosis-Müller muscle complex surgery from fixation of the skin and muscle to the tarsal plate. If the fold disappears despite the ptosis being well corrected, the fold can be created using the nonincision method in revisional surgery. ${ }^{12,20,21}$

\section{Asymmetry}

Asymmetry, the most common complication, is mostly caused by bleeding or swelling during surgery or by noncooperation of the patient. Additionally, unskilled surgeons sometimes fix anatomically different structures. Asymmetry must be corrected as soon as possible, ideally within 3 weeks after the first surgery. ${ }^{12,20,21}$

\section{Lagophthalmos}

Undercorrection is better than making lagophthalmos, which is mostly caused by an incomplete release of fibrosis tissue. If the eyes are symmetrical, a slight undercorrection looks attractive. When lagophthalmos is still noted at 2 to 3 weeks after operation, reoperation should be considered. ${ }^{13,23}$

\section{Cornea Injury}

Because of exposure of the thread toward the conjunctiva during fixation to the tarsal plate, a long duration of dryness during surgery, or irregularity in the conjunctiva after levator
aponeurosis-Müller muscle complex advancement, the eyes feel uncomfortable and injury is possible. ${ }^{22,23}$

If thread exposure is found when the eyelid is turned inside out, it is removed or corrected, artificial tears and an antibiotic eye drop are prescribed, and a contact lens is prescribed to protect the cornea from further pain, conjunctival swelling is reduced, and the patient becomes comfortable. $^{22,23}$

\section{Brow Ptosis}

Patients with subclinical ptosis tend to have high brows. If the levator aponeurosis-Müller muscle complex is corrected, the brow is no longer involved in raising the upper eyelids, so its position moves downward by approximately 3 to $4 \mathrm{~mm}$ from the preoperative position. In this case, the look of patients whose eyes and eyebrows become closer becomes serious and the lateral side of the sub-brow area thickens, which causes the swollen feeling to persist. In this case, a browpexy by incision or an endoscopic forehead brow lift is recommended. ${ }^{24}$

\section{Conclusion}

We have described various methods for creation of bigger and brighter eyes, which used to be thought of as just the creation of the double lid crease, the larger the better. The current thought for Asian upper blepharoplasty is not so much the formation of a double crease and excessive focus of how high the crease should be placed. The look that Asian patients strive for now is natural, beautifully enhanced Asian eyes that brighten the face and bring attention of the viewer to the eyes, one of the most, if not the most important component of an attractive face. As such, the attention is placed on enlargement of the palpebral aperture and harmonizing the eye measurements with the rest of the facial proportions. In many cases, there is subclinical ptosis, and the purpose of an Asian blepharoplasty is to improve this ptosis through any available means. A nonincision approach, a partial incision approach, as well as an open incision approach will all be able to improve the subclinical ptosis, while also contributing in the formation of a crease.

\section{References}

1 Chen WPD. Asian blepharoplasty and the eyelid crease. 2nd ed. New York, NY: Elsevier; 2006:2-22

2 Lee EI, et al. The relationship between eyebrow elevation and height of the palpebral fissure: should postoperative brow descent be taken into consideration when determining the amount of blepharoptosis correction? Arch Aesthet Plast Surg 2014;20(1): 20-25

3 Doxanas MT, Anderson RL. Oriental eyelids. An anatomic study. Arch Ophthalmol 1984;102(8):1232-1235

4 Yuzuriha S, Matsuo K, Kushima H. An anatomical structure which results in puffiness of the upper eyelid and a narrow palpebral fissure in the Mongoloid eye. Br J Plast Surg 2000;53(6):466-472

5 Kim MK, Rathbun JE, Aguilar GL, Seiff SR. Ptosis surgery in the Asian eyelid. Ophthal Plast Reconstr Surg 1989;5(2):118-126 
6 Perrett DI, May KA, Yoshikawa S. Facial shape and judgements of female attractiveness. Nature 1994;368(6468):239-242

7 Mehta HK. The contralateral upper eyelid in ptosis: some observations pertinent to ptosis corrective surgery. $\mathrm{Br} \mathrm{J}$ Ophthalmol 1979;63(2):120-124

8 Jones LT, Quickert MH, Wobig JL. The cure of ptosis by aponeurotic repair. Arch Ophthalmol 1975;93(8):629-634

9 Lee EI, Ahn TJ. Mild ptosis correction with the stitch method during incisional double fold formation. Arch Plast Surg 2014;41(1):71-76

10 Liu D, Hsu WM. Oriental eyelids. Anatomic difference and surgical consideration. Ophthal Plast Reconstr Surg 1986;2(2):59-64

11 Hwang K, Kim DJ, Chung RS, Lee SI, Hiraga Y. An anatomical study of the junction of the orbital septum and the levator aponeurosis in Orientals. Br J Plast Surg 1998;51(8):594-598

12 Weng CJ. Oriental upper blepharoplasty. Semin Plast Surg 2009; 23(1):5-15

13 Ji Y, Qian Z, Dong Y, Zhou H, Fan X. Quantitative morphometry of the orbit in Chinese adults based on a three-dimensional reconstruction method. J Anat 2010;217(5):501-506

14 Bour LJ, Aramideh M, de Visser BW. Neurophysiological aspects of eye and eyelid movements during blinking in humans. J Neurophysiol 2000;83(1):166-176

15 Ahn TJ. Blepharoptosis correction with stitch method. J Korean Soc Aesthetic Plast Surg 2010;16:167-170
16 Wheatcroft SM, Vardy SJ, Tyers AG. Complications of fascia lata harvesting for ptosis surgery. $\mathrm{Br} \mathrm{J}$ Ophthalmol 1997;81(7): 581-583

17 Ahn TJ, Kim KK. Cases of mild ptosis correction with suturemethod. Arch Aesthetic Plast Surg 2012;18:15-20

18 Chang SH, Chen WP, In Chang C, Ahn TJ. Comprehensive review of Asian cosmetic upper eyelid oculoplastic surgery: Asian blepharoplasty and the LikeArch. Aesthetic Plast Surg 2014;20(3): 129-139

19 Shimizu Y, Nagasao T, Asou T. A new non-incisional correction method for blepharoptosis. J Plast Reconstr Aesthet Surg 2010; 63(12):2004-2012

20 Sayoc BT. Surgery of the Oriental eyelid. Clin Plast Surg 1974;1(1): 157-171

21 Hin LC. Oriental blepharoplasty-a critical review of technique and potential hazards. Ann Plast Surg 1981;7(5):362-374

22 Oestreicher J, Mehta S. Complications of blepharoplasty: prevention and management. Plast Surg Int 2012;2012: 252368

23 Newman NJ. Perioperative visual loss after nonocular surgeries. Am J Ophthalmol 2008;145(4):604-610

24 Lee JM, Lee TE, Lee H, Park M, Baek S. Change in brow position after upper blepharoplasty or levator advancement. J Craniofac Surg 2012;23(2):434-436 